\title{
Derivation of sarcomas from mesenchymal stem cells via inactivation of the Wnt pathway
}

\author{
Igor Matushansky, ${ }^{1}$ Eva Hernando, ${ }^{2}$ Nicholas D. Socci, ${ }^{3}$ Joslyn E. Mills, ${ }^{4}$ Tulio A. Matos, ${ }^{4}$ \\ Mark A. Edgar, ${ }^{5}$ Samuel Singer, ${ }^{6}$ Robert G. Maki, 7 and Carlos Cordon-Cardo ${ }^{4}$ \\ 1Department of Medicine, Columbia University, New York, New York, USA. 'Department of Pathology, New York University School of Medicine, \\ New York, New York, USA. ${ }^{3}$ Computational Biology Center, Memorial Sloan-Kettering Cancer Center (MSKCC), New York, New York, USA. \\ ${ }^{4}$ Department of Pathology, Columbia University, New York, New York, USA. ${ }^{5}$ Department of Pathology, \\ ${ }^{6}$ Department of Surgery, and 7 Department of Medicine, MSKCC, New York, New York, USA.
}

\begin{abstract}
Malignant fibrous histiocytoma (MFH), now termed high-grade undifferentiated pleomorphic sarcoma, is a commonly diagnosed mesenchymal tumor, yet both the underlying molecular mechanisms of tumorigenesis and cell of origin remain unidentified. We present evidence demonstrating that human mesenchymal stem cells (hMSCs) are the progenitors of MFH. DKK1, a Wnt inhibitor and mediator of hMSC proliferation, is overexpressed in MFH. Using recombinant proteins, antibody depletion, and siRNA knockdown strategies of specific Wnt elements, we show that DKK1 inhibits hMSC commitment to differentiation via Wnt2/ $\beta$-catenin canonical signaling and that $\mathrm{Wnt} 5 \mathrm{a} / \mathrm{JNK}$ noncanonical signaling regulates a viability checkpoint independent of Dkk1. Finally, we illustrate that hMSCs can be transformed via inhibition of Wnt signaling to form MFHlike tumors in nude mice, and conversely, MFH cells in which Wnt signaling is appropriately reestablished can differentiate along mature connective tissue lineages. Our results provide mechanistic insights regarding the cell of origin of MFH, establish what we believe is a novel tumor suppressor role for Wnt signaling, and identify a potential therapeutic differentiation strategy for sarcomas.
\end{abstract}

\section{Introduction}

The essence and origin of malignant fibrous histiocytoma (MFH) have been debated for over 40 years. It was characterized as a morphologically unique soft tissue sarcoma subtype in 1963 (1), and it was said at the time that "the issue of histogenesis [of MFH] is largely unresolvable" (2). More recently it was stated that "MFH is now regarded as synonymous with [high-grade] undifferentiated pleomorphic sarcoma and essentially represents a diagnosis of exclusion" (3). Yet MFH, now termed [high-grade] undifferentiated pleomorphic sarcoma, has historically accounted for approximately $25 \%$ of all patients accrued to sarcoma clinical trials $(4,5)$. While its diagnosis may have declined in recent years, it remains a recognized and distinct sarcoma subtype without effective treatment options for metastatic disease. Therefore, understanding its histogenesis and discerning its molecular features are critical steps in identifying relevant targets for therapeutic intervention.

In the present study, we postulate that MFH represents a transformed adult human mesenchymal stem cell (hMSC). To validate our hypothesis, we use a well-characterized set of genes representative of the stem cell phenotype to establish an association between MFH and hMSCs. Furthermore, we hypothesized that if hMSCs are the cells of origin of MFH, then genes differentially expressed in $\mathrm{MFH}$ as compared with other sarcomas should be relevant to hMSC biology. Gene expression and related functional analyses based on the latter concept led us to unravel the oncogenic role of DKK1 in hMSC transformation. Dkk1 is a secreted inhibitor of the

Nonstandard abbreviations used: ADM, adipocytic differentiation medium; EMofMD, early markers of mesenchymal differentiation; hMSC, human mesenchymal stem cell; hrDKK1, human recombinant DKK1; IHC, immunohistochemistry; LS, liposarcoma(s); MFH, malignant fibrous histiocytoma; ODM, osteogenic differentiation medium; rWnt2, recombinant Wnt2; STS, soft-tissue sarcoma(s).

Conflict of interest: The authors have declared that no conflict of interest exists. Citation for this article: J. Clin. Invest. 117:3248-3257 (2007). doi:10.1172/JCI31377. highly conserved Wnt developmental program (6) and a protein that has been previously shown to be necessary for proliferation of hMSCs (7). Although the relevance of Wnt signaling in epithelial malignancies has been well established (reviewed in ref. 8), its role in mesenchymal tumors remains mainly unexplored.

\section{Results}

MFH expression patterns correlate with those of hMSCs. To explore the possibility that MFH derives from the transformation of hMSCs, RNA was isolated from proliferating hMSCs, hybridized on Affymetrix U133a microarrays, and compared with previously profiled sarcomas (9). Soft-tissue sarcomas (STS) define a group of histologically and genetically diverse tumors of mesenchymal origin, with greater similarity within a given subtype than with cultured hMSCs. For this reason, we used a well-characterized panel of stem cell-specific genes $(10,11)$ as a discriminant to evaluate the potential relationship between hMSCs, MFH, and other sarcoma subtypes. Unsupervised hierarchical clustering analysis using such a stem cell gene signature revealed that hMSCs are significantly associated exclusively with MFH (Figure 1A). Previous transcriptional pairwise analysis had also revealed the association between hMSCs and MFH (12). Using an in vitro approach in which hMSCs are differentiated into prototypic connective cells (i.e., fat and bone) and gene expression analysis performed (via Affymetrix U133a arrays) at multiple temporal points during differentiation, we found that MFH exhibits the closest association among the differentiating cells to undifferentiated hMSCs. Similarly, committed (i.e., lineage-specific) sarcoma cell lines (i.e., LS141 liposarcoma [LS] and SAOS2 osteosarcoma) associate with hMSCs, differentiating into either adipocytes or osteocytes, respectively, independently corroborating the hMSCsarcoma association (Supplemental Figure 1 and Supplemental Text; supplemental material available online with this article; doi:10.1172/JCI31377DS1). 
A

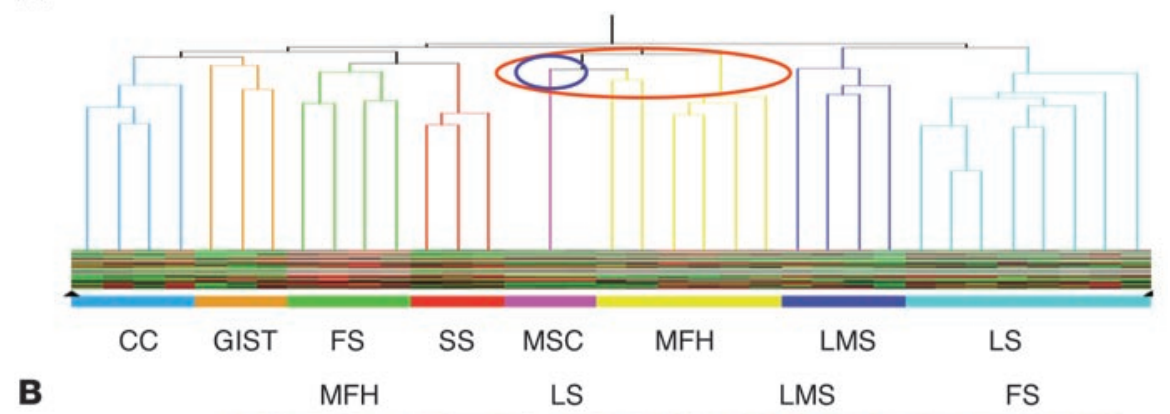

\section{Figure 1}

MFH associated with hMSCs. (A) Unsupervised hierarchical clustering of a panel of STS (described in detail in ref. 9) using a stem cell gene list (Supplemental Table 1). (B) Immunohistochemical staining of differentiation-associated proteins on the indicated tumor specimens from a tissue microarray. A single stain representative of 10 tissue samples each of MFH, LS, and leiomyosarcomas (LMS), and of 6 tissue samples each of fibrosarcomas (FS) are shown. Original magnification, $\times 200$.

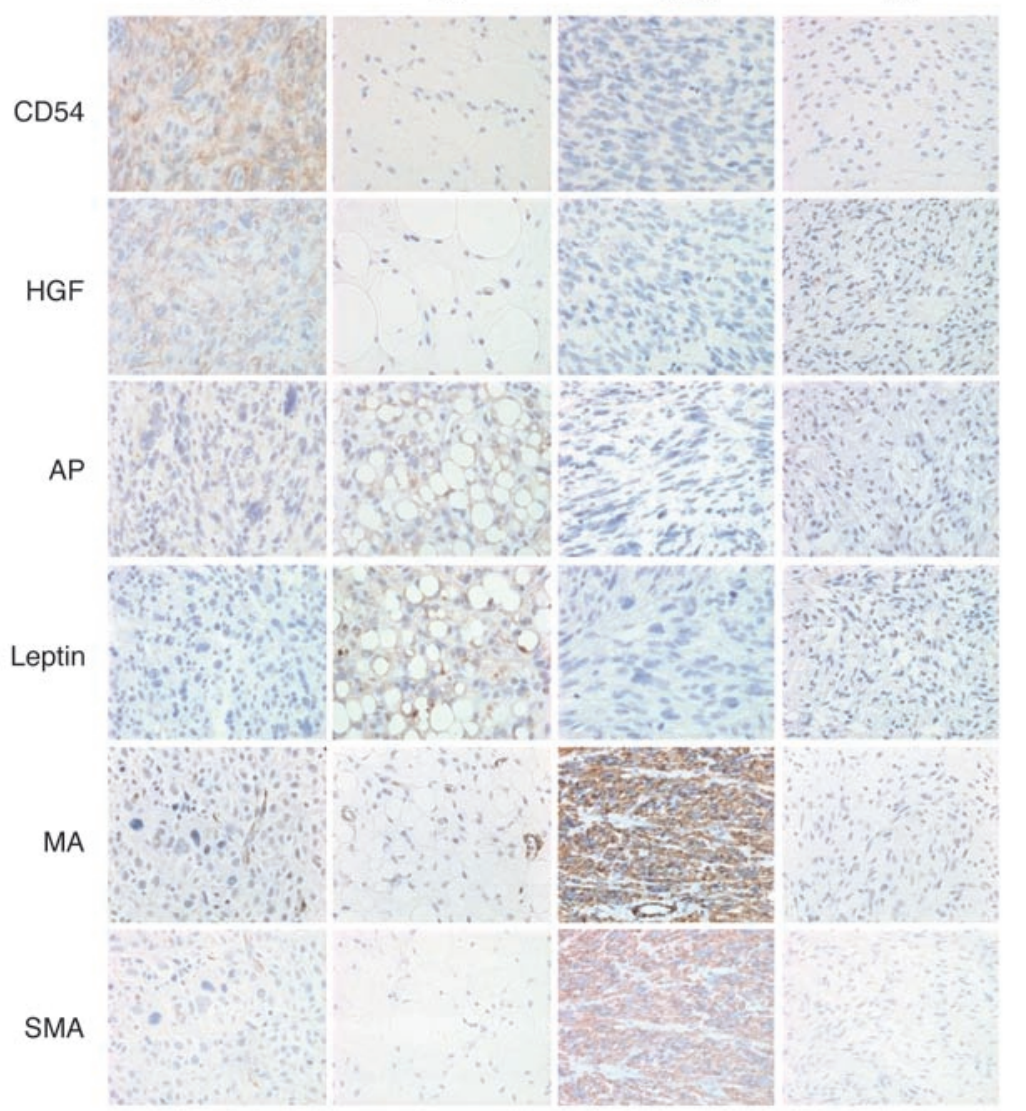

To further establish the specific relationship between sarcoma subtypes and their lineage of origin, we performed immunohistochemical analysis of lineage-specific markers on STS in a tissue microarray. As illustrated in Figure 1B, staining these tumors for fat-specific markers (i.e., leptin and adiponectin) resulted in intense immunostaining of LS only. As expected, only leiomyosarcomas (LMSs) strongly expressed muscle-specific markers (i.e., muscle actin and $\alpha$-smooth muscle actin). Similarly, when the same lesions were studied with MSC markers (e.g., CD54 and HGF) (13), we observed that only MFH cases displayed a positive phenotype, reinforcing its putative MSC origin. As a negative control, low-grade fibrosarcomas exhibited negative phenotypes for all biomarkers analyzed (Figure 1B). Although there are other classical MSC markers used for FACS analysis, they are not suited for our approach of immunohistochemistry (IHC) on sarcoma tissue (13). Additionally, we were specifically interested in hMSC markers that were not only present and/or specific in hMSCs but also decreased their expression as hMSCs differentiated. Accord- ingly, we screened all known mesenchymal markers against our differentiation gene expression data to determine which, if any, decrease with differentiation (data not shown). Of all the potential MSC markers, we found that CD54 and HGF were the best candidates, and they were therefore used in this study.

$D K K 1$ is overexpressed and Wnt/ $\beta$-catenin activity is absent in MFH cells. Previous analysis of gene expression profiles by our laboratory (9) and others (14) has led us to observe that genes overexpressed in specific sarcoma subtypes are representative of the cell/lineage of origin of that particular neoplasm (Figure 2A and Supplemental Text). We hypothesized that if hMSCs are the cells of origin of MFH, then genes overexpressed in $\mathrm{MFH}$ as compared with other sarcoma subtypes should be relevant to hMSC biology and function. ANOVA on gene expression profiles of the panel of STS stratified by genes overexpressed in MFH (Figure 2A) identified many structural proteins and/or proteins whose functions are not well established. The most prominent exception was DKK1, a secreted inhibitor of the 
A

$\begin{array}{lccccccc} & \text { CC } & \text { SS } & \text { FS } & \text { GIST } & \text { MFH } & \text { LMS } & \text { LS } \\ \text { HAS1 } & 0.83 & 0.69 & 0.89 & 0.58 & 28.96 & 0.92 & 1.72 \\ \text { TNXB } & 0.62 & 0.69 & 0.77 & 0.85 & 28.11 & 1.64 & 1.15 \\ \text { MFAP5 } & 0.90 & 0.27 & 0.11 & 0.04 & 19.55 & 4.54 & 1.29 \\ \text { DKK1 } & 0.50 & 0.77 & 7.81 & 0.58 & 18.20 & 1.18 & 0.80 \\ \text { TNA } & 0.30 & 0.91 & 0.49 & 0.64 & 12.27 & 0.80 & 1.09 \\ \text { BF } & 1.61 & 0.90 & 0.64 & 5.73 & 12.24 & 3.05 & 0.29 \\ \text { TNFAIP6 } & 0.74 & 0.25 & 1.33 & 0.49 & 11.90 & 1.37 & 0.69 \\ \text { S100A4 } & 1.43 & 1.05 & 1.31 & 0.41 & 11.45 & 1.21 & 0.37 \\ \text { DCN } & 1.72 & 0.67 & 1.50 & 0.16 & 11.10 & 0.58 & 0.48 \\ \text { FBLN2 } & 0.81 & 2.89 & 0.72 & 0.10 & 10.63 & 0.36 & 0.66\end{array}$

C

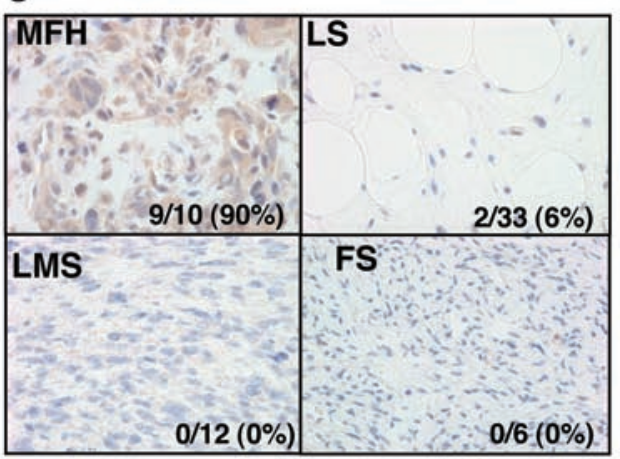

E

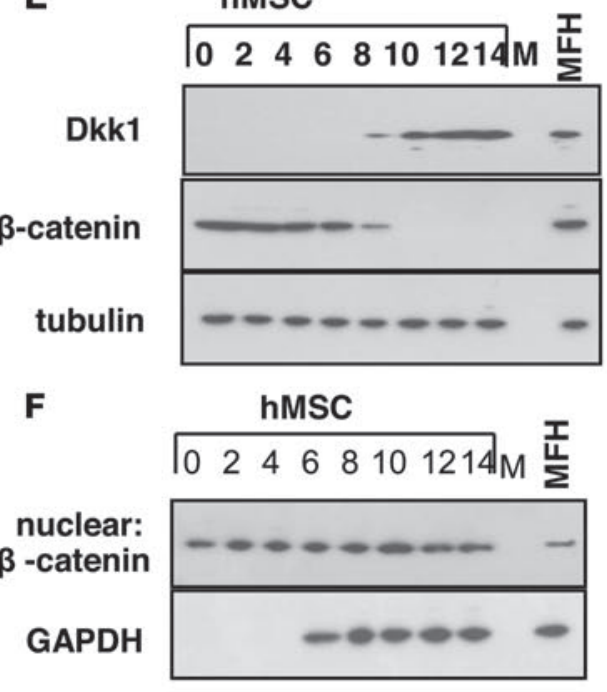

B

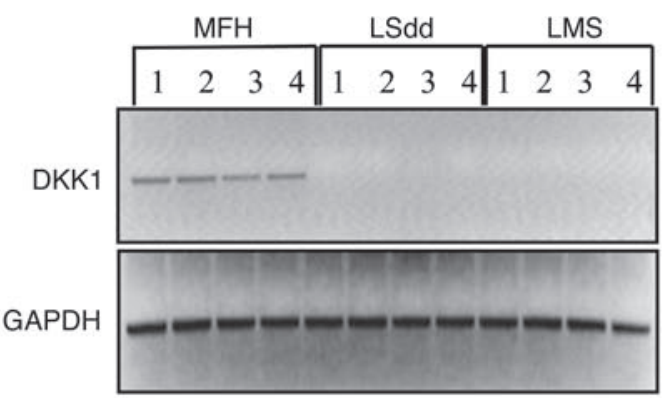

D

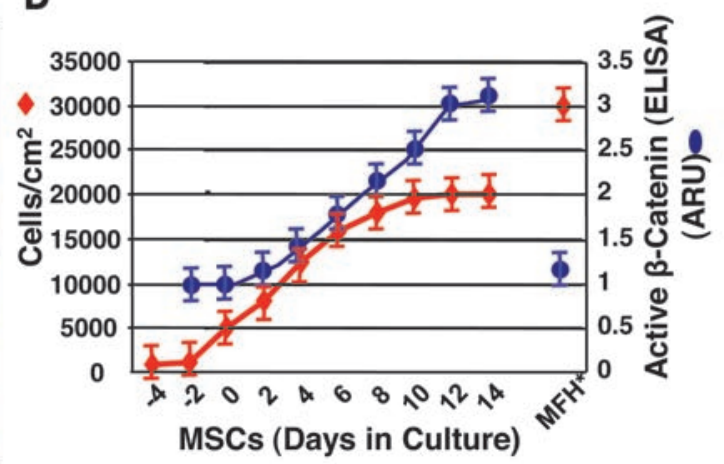

G
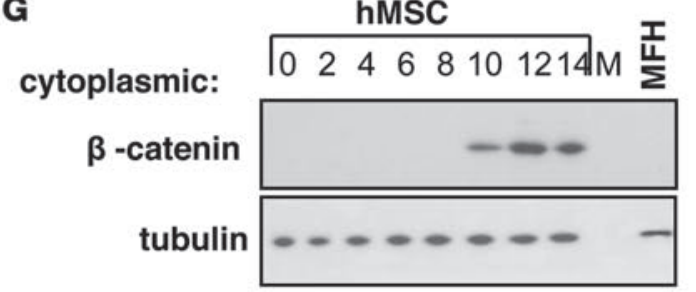

H

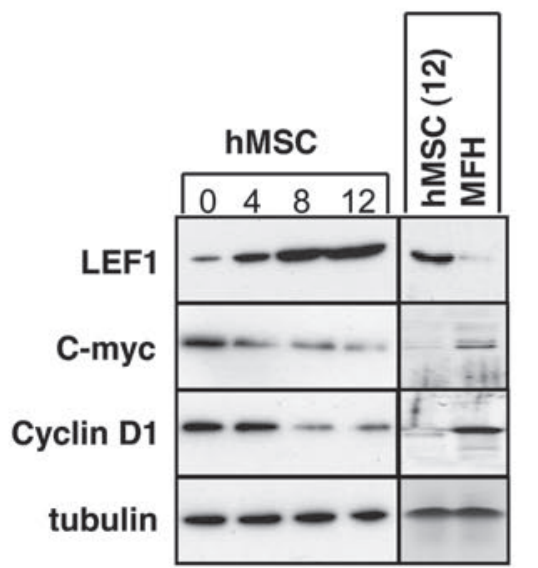

Figure 2

DKK1 and $\beta$-catenin patterns in hMSCs and MFH.

(A) ANOVA of gene expression analysis of the indicated sarcomas listed in order of genes overexpressed in MFH. (B) DKK1 levels by RT-PCR in the indicated sarcomas. (C) Dkk1 expression by IHC staining in the indicated sarcomas and percentage of samples expressing intense staining. Original magnification, $\times 200$. (D) Cell density of hMSCs proliferating in vitro in relation to $\beta$-catenin activity. Immunoblot analysis of Dkk1 and $\beta$-catenin in total cellular extracts $(\mathbf{E})$, nuclear extracts, $(\mathbf{F})$ and cytoplasmic extracts $(\mathbf{G})$ in the indicated samples. Immunoblot analysis of LEF1, c-myc, and cyclin D1 tubulin $(\mathbf{H})$ in the indicated samples. highly conserved Wnt developmental program (6) known to be necessary for proliferation of hMSCs (7). Immunohistochemical studies for DKK1 on a panel of STS (Figure 2C) and RT-PCR (Figure 2B) analysis of DKK1 transcripts on RNA isolated from fresh-frozen MFHs, LS, and leiomyosarcomas demonstrated that DKK1 is more significantly expressed in MFH than other sarcoma subtypes.

Having identified DKK1 as a protein expressed by both $\mathrm{MFH}$ and hMSCs, we next sought to examine the patterns of DKK1 and $\beta$-catenin (the effector arm of the Wnt-canonical pathway; ref. 8) expression in hMSCs in relation to MFH cells. Prockop et al. $(7,15)$ established that expression of DKK1 closely parallels proliferation of
hMSCs in vitro and that Dkk1 promotes reentry of hMSCs into the cell cycle. To examine the relationship between the levels of DKK1 in hMSCs and MFH, hMSCs were grown as previously described (7). Cell density was measured at the indicated time points (Figure 2E), and total cellular protein was isolated from hMSCs at an initial cell density of approximately $1 \times 10^{5}$ cells $/ \mathrm{cm}^{2}$. Immunoblot analysis of DKK1 and $\beta$-catenin as a function of proliferation indicated that DKK1 is expressed specifically during the proliferative phase of hMSCs while $\beta$-catenin is expressed in an inverse pattern - absent during proliferation and present during the stationary phase (Figure 2E). In comparison, the MFH0022 cell line (assayed at confluence; other 
A

A

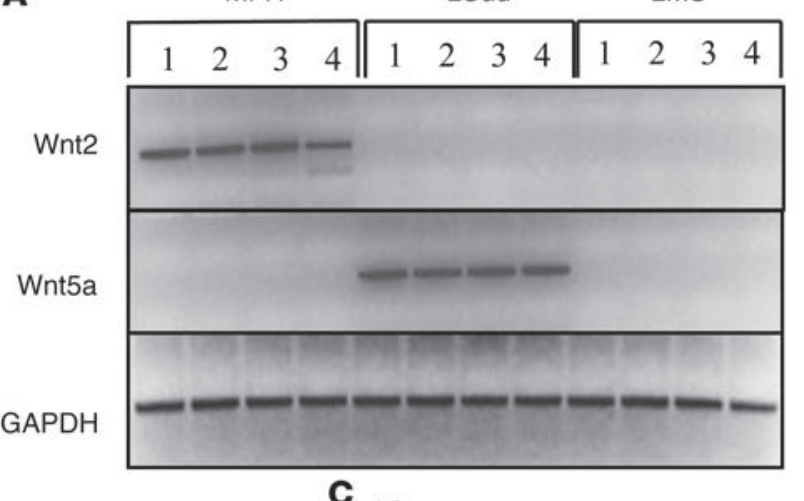

B

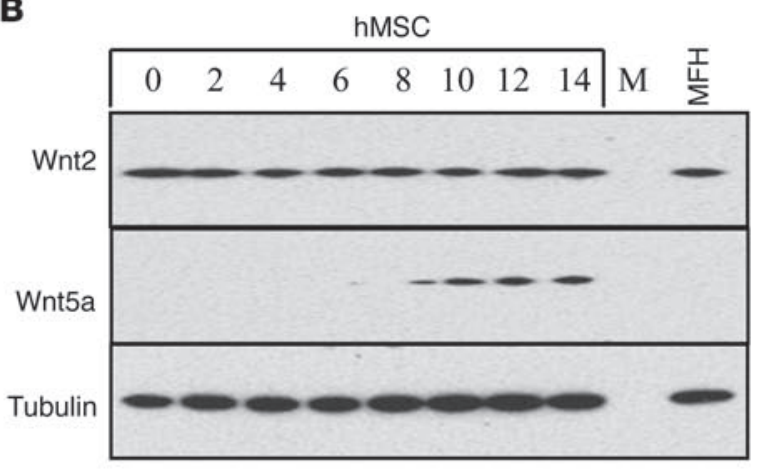

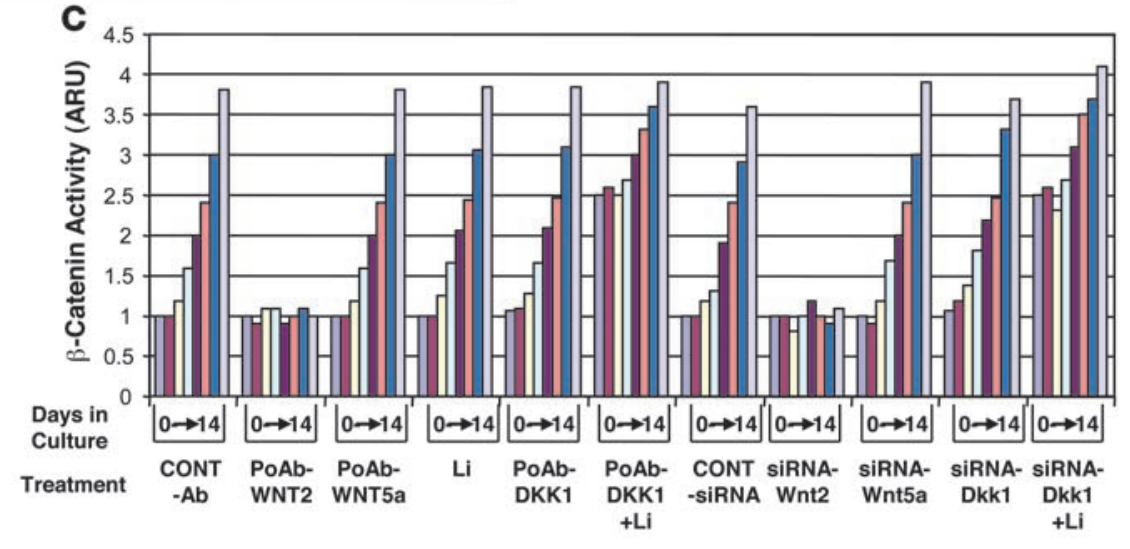

Figure 3

Wnt signaling in hMSCs. (A) Expression of Wnt2 and Wnt5a by RT-PCR in the indicated sarcomas. (B) Immunoblot analysis of Wnt2 and Wnt5a in the indicated samples. (C) ELISA of $\beta$-catenin in the indicated samples following specified treatments.

MFH culture time points showed similar pattern to confluence; data not shown) displayed both Dkk1 and $\beta$-catenin (Figure 2E), paralleling the pattern observed in proliferating hMSCs (in particular the day 8 time point) and suggesting that MFH arises specifically from the proliferating hMSC population.

Since $\beta$-catenin levels are primarily regulated by cytoplasmicto-nuclear shuttling (8), we examined the levels of nuclear and cytoplasmic $\beta$-catenin in both proliferating hMSCs and MFH. As hMSCs transitioned from the proliferating to the stationary phase, $\beta$-catenin accumulated first in the cytoplasm (Figure $2 \mathrm{G}$ ) and then moved into the nucleus (Figure 2F). In contrast, while confluent and exponentially growing MFH cells expressed cytoplasmic $\beta$-catenin, no nuclear $\beta$-catenin was detectable (Figure 2, F and G). Finally, active $\beta$-catenin levels (via ELISA for the active form of $\beta$-catenin) measured as a function of time paralleled the nuclear $\beta$-catenin immunoblot pattern, with accumulation of $\beta$-catenin observed as hMSCs ceased proliferating and no measurable expression in MFH (see Figure 2E). Additionally, induction of $\beta$-catenin levels via combined treatment of MFH cells with lithium (an activator of $\beta$-catenin via inhibition of the Wnt pathway intermediate inhibitor GSK-3 $\beta$ ) and inhibition of Dkk1 protein or RNA expression using monoclonal anti-Dkk1 antibody or siRNADkk1 resulted in increased total and nuclear $\beta$-catenin accumulation (Supplemental Figure 2 and Supplemental Text).

Finally, we examined the expression of 3 known $\beta$-catenin target genes in proliferating and stationary hMSCs as well as in MFH cells. As shown in Figure 2H (left panel), LEF1 levels increased with increasing levels of $\beta$-catenin while c-myc and cyclin D1 levels decreased with increasing $\beta$-catenin levels. We also used chromatin immunoprecipitation to demonstrate that $\beta$-catenin directly binds the promoters of myc and cyclin D1 and that the promoter exists in a primed repressive state (Supplemental Text and Supplemental Figures 3 and 4). Furthermore, the decrease in c-myc and cyclin D1 was consistent with the observed loss of hMSC proliferation (Figure 2E). On the other hand, as shown in Figure $2 \mathrm{H}$ (right panel), MFH cells (assayed at confluence) expressed higher levels of c-myc and cyclin D1 than hMSCs (assayed at confluence; day 12). The expression of c-myc and cyclin D1 in MFH cells at confluence may explain their continuous proliferation. Additionally, the low levels of LEF1 in MFH confirmed the lack of Wnt signaling as inferred from lack of active (Figure 2E) and nuclear $\beta$-catenin (Figure $2 \mathrm{~F}$ ).

Taken together, these results suggest that MFH cells fail to accumulate nuclear $\beta$-catenin due to limited endogenous production, as fitting an hMSC transformed during their proliferative state where endogenous hMSC $\beta$-catenin protein levels were naturally low (Figure 2F).

Wnt2-canonical signaling in hMSCs mediates commitment to differentiation. Since nuclear $\beta$-catenin levels increased as hMSCs reached confluence and since no nuclear $\beta$-catenin was detectable in MFH cells, we sought to compare the patterns of the main determinants of $\beta$-catenin activity (the Wnt proteins) in hMSCs and MFH. It has been reported that Wnt2, Wnt4, Wnt5a, Wnt11, and Wnt16 are detectable in hMSCs while the majority of other Wnt products are not (16). To limit our exploration to the Wnt genes relevant to MFH 
A

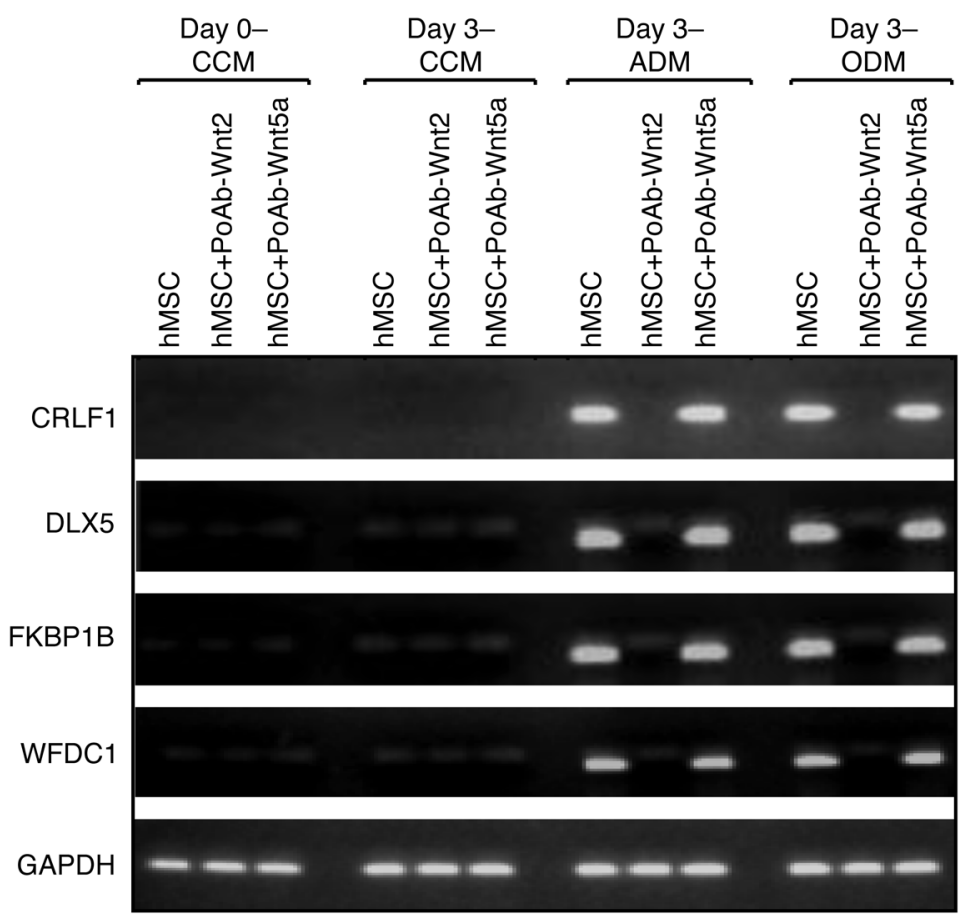

B
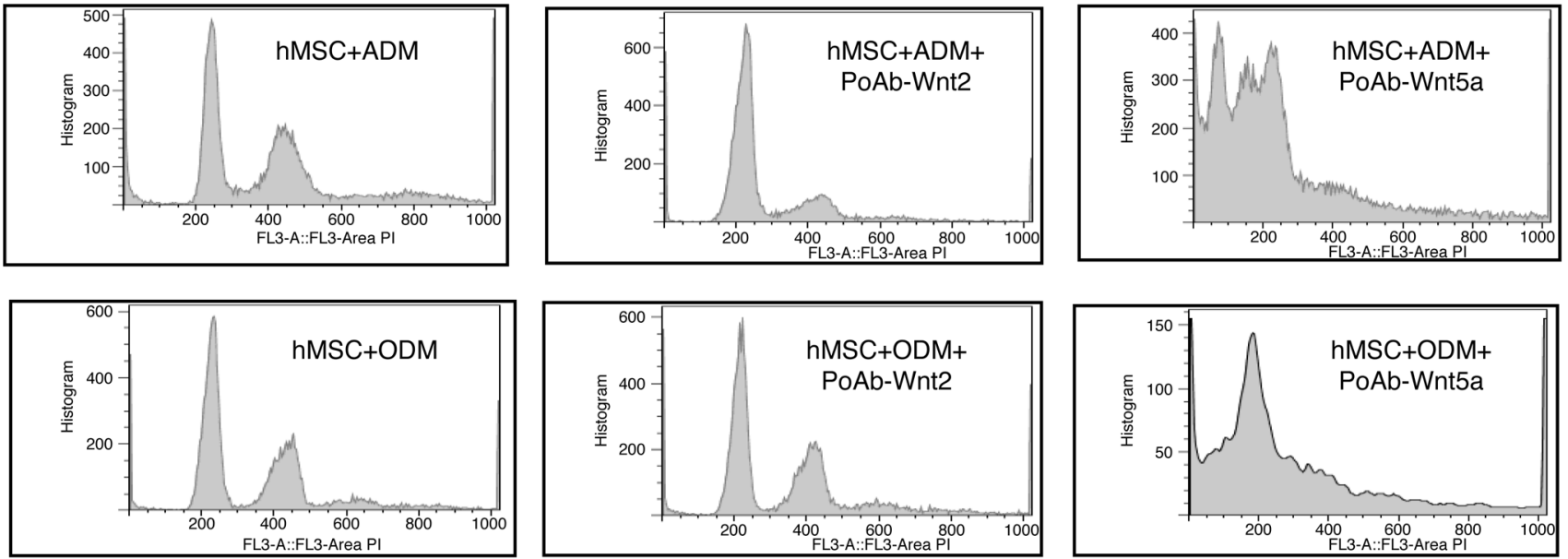

Figure 4

Wnt5a regulates a commitment viability checkpoint. (A) Expression of EMofMD by RT-PCR in hMSCs grown in either continuous culture medium (CCM), ADM, or ODM, as indicated. (B) Cell cycle analysis of hMSCs after 7 days of culture under the specified treatments.

as transformed hMSCs, we examined the relative expression of Wnt proteins in MFH as compared with other sarcoma subtypes (Supplemental Table 2). Of the 5 Wnts referred to above, Wnt2 was highly overexpressed in MFH and Wnt5a was absent as compared with other sarcoma subtypes. These results were confirmed in a panel of STS by RT-PCR (Figure 3A). Additionally, during in vitro hMSC proliferation, Wnt5a protein accumulated as cells ceased to grow (in agreement with previously published RNA patterns; ref. 15) while Wnt2 levels stayed constant (Figure 3B). Given these observations, 2 possibilities may explain the mechanism of $\beta$-catenin accumulation: (a) Wnt5a accumulation results in canonical Wnt pathway activation and $\beta$-catenin nuclear accumulation; or (b) Wnt 2 activation results in constitutive $\beta$-catenin activation, which is repressed by Dkk1, and thus $\beta$-catenin only accumulates when Dkk1 levels decrease. To explore these possibilities, total cellular protein was isolated from hMSCs at the indicated time points of in vitro proliferation after treatment with either a polyclonal antibody or with siRNA against either Wnt2 or Wnt5a under the indicated conditions (see Supplemental Figure 2 and Supplemental Text regarding the specificity of siRNA-Wnt2 and siRNa-Wnt5a) and assayed via ELISA for active $\beta$-catenin (Figure $3 \mathrm{C}$ ). Only the use of either blocking antibody PoAb-Wnt2 or siRNA-Wnt2 was specifically able to suppress active $\beta$-catenin accumulation, not PoAb-Wnt5a or siRNA-Wnt5a. These data suggest that Wnt2 mediates signaling via canonical (Wnt/ß-catenin) pathway in hMSCs, controlled by Dkk1 levels.

Wnt2 mediates commitment while Wnt5a mediates a viability checkpoint in hMSCs. To further explore the role of Wnt2 signaling in hMSC differentiation, hMSCs were pretreated with either PoAb-Wnt2 or 

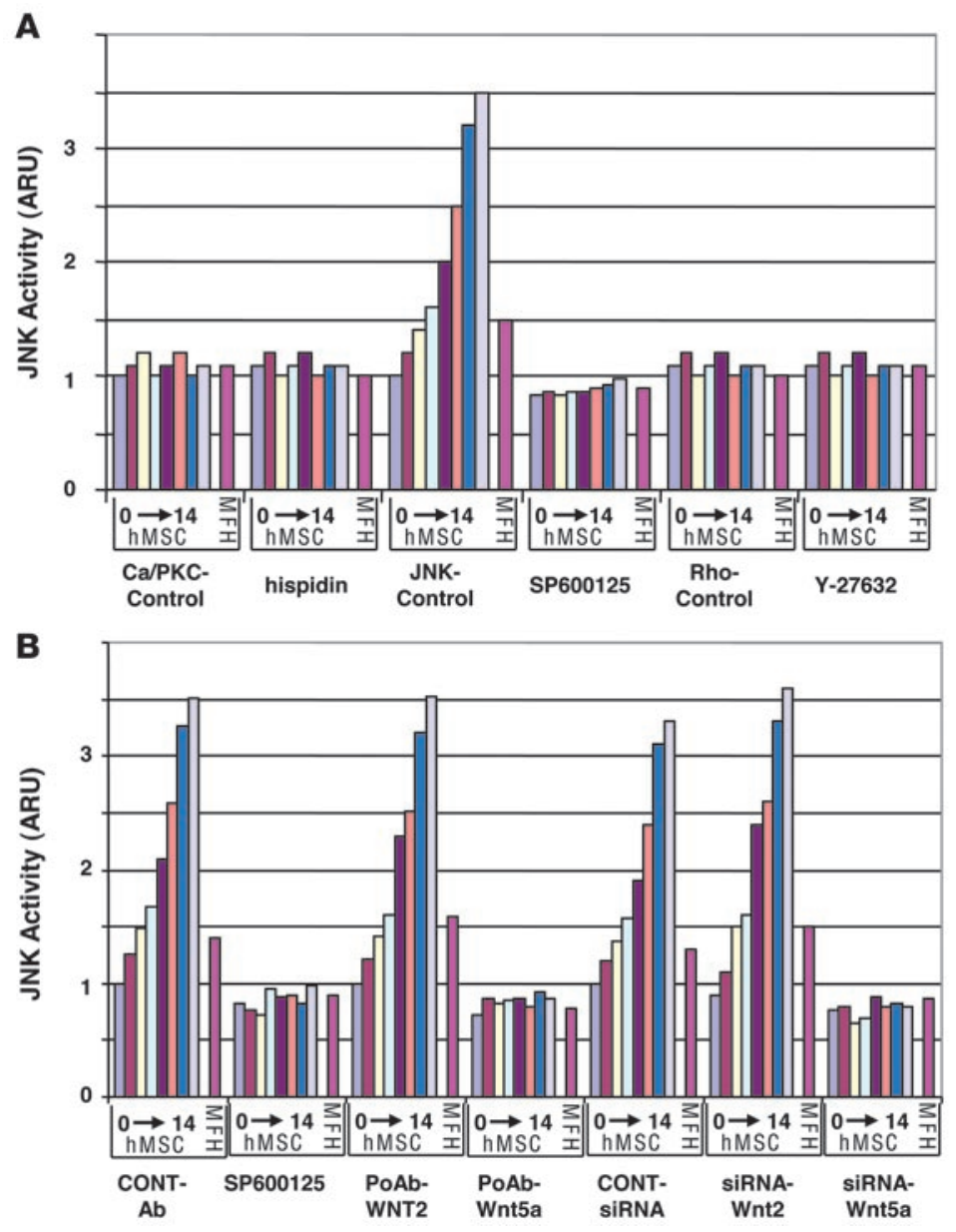

Figure 5

Wnt5a signals through Jnk. (A) Relative activity of Wnt noncanonical signaling and its inhibitors: Wnt/Ca pathway (Ca and hispidin), Wnt/Rho pathway (Rho and Y-276632), and Wnt/Jnk (JNK and SP600125) in specified samples under the indicated conditions. (B) Relative activity of Wnt/Jnk in specified samples under the indicated conditions. All measurements ( $\mathbf{A}$ and $\mathbf{B})$ indicated are the average of 2 independent time series.

PoAb-Wnt5a, then induced to differentiate via further treatment with adipocytic differentiation medium (ADM) or osteogenic differentiation medium (ODM), and finally assessed for early markers of mesenchymal differentiation (EMofMD) (Figure 4A) (see Supplemental Figure 5 and Supplemental Text for further details). Treatment of cells with PoAb-Wnt2 but not PoAb-Wnt5a led to the inability of hMSCs to express EMofMD, suggesting that Wnt2/ $\beta$-catenin activity is necessary for commitment to the mesenchymal differentiation program. (In the Supplemental Text and in Supplemental Figure 5, we discuss and illustrate that MFH cells treated with lithium and PoAbDkk1 [which we show results in nuclear $\beta$-catenin accumulation; Supplemental Figure 2] display detectable expression of EMofMD.)

In contrast, hMSCs pretreated with PoAb-Wnt5a express EMofMD (Figure 4A), suggesting that Wnt5a does not affect commitment of hMSCs to differentiation. However, further growth of hMSCs following PoAb-Wnt5a treatment resulted in apoptotic-appearing cells as compared with control cells (Figure 4B). Propidium iodide staining and DNA content detection demonstrated an accumulation of a large sub-G1 peak consistent with apoptosis (Figure 4B). This was not observed in either hMSCs proliferating as controls or in hMSCs treated with PoAb-Wnt2. Based on this set of observations, we concluded that Wnt2 (via Wnt/ $\beta$-catenin) signaling is necessary for commitment to differentiation while Wnt5a signaling does not promote commitment to differentiation (as judged by expression of EMofMD) but may mediate an antiapoptotic signal.

Wnt5 a signals through JNK in bMSCs. Since Wnt5a has been previously reported to mediate noncanonical Wnt signaling, we sought to examine the main noncanonical Wnt pathways active during hMSC proliferation and MFH sarcomagenesis. As discussed in a previous review (17), Wnt/noncanonical is mediated by 3 main pathways: WntPKC/calmodulin, Wnt-Rho GTPase (Rho/ROK/cdd42), and $\mathrm{Wnt} / \mathrm{JNK}$. Total cellular protein was isolated at multiple time points during hMSC in vitro proliferation and in confluent MFH cells after continuous culturing both in the absence and presence of an inhibitor to each pathway (hispidin, inhibitor of PKC, ref. 18; Y-27632, inhibitor of ROK, ref. 19; SP600125, inhibitor of JNK, ref. 20). The activity of each pathway was assayed as described in Methods. Only Wnt/JNK changed during hMSC in vitro proliferation (Figure 5A), increasing approximately 4-fold as compared with baseline activity at plating. This activity was specifically blocked by JNK inhibitor SP600125.

To assess whether or not the rise in Wnt/JNK activity was Wnt5a mediated, Wnt/JNK signaling activity was measured in proliferating hMSCs either transfected with siRNA-Wnt5a or grown in medium supplemented with PoAb-Wnt5a. Under these conditions, no Wnt/JNK accumulation was observed, similar to the pattern of hMSCs grown in media supplemented with SP600125. As a control, hMSCs transfected with siRNA-Wnt2 or grown in medium supplemented with PoAb-Wnt2 showed no effect on Wnt/JNK activity (Figure 5B). These studies demonstrate that Wnt5a accumulation during hMSC growth activates Wnt/JNK activity, a well-characterized antiapoptotic pathway (21). In contrast, MFH cells at confluence do not show significant Wnt/JNK activity (Figure 5, A and B), mirroring the pattern seen when comparing $\beta$-catenin activity in MFH and hMSCs (Figure 3, D and E).

Recapitulation of hMSC Wnt signaling patterns in MFH cells leads to controlled differentiation of MFH cells into mature connective tissue lineages. We have shown that Dkk 1 is overexpressed in MFH and that it controls a canonical Wnt2 signaling pathway required for commitment to mesenchymal differentiation while Wnt5a/JNK noncanonical signaling regulates a commitment-viability checkpoint. Based on these findings, we sought to further investigate whether establishing both Wnt2/canonical signaling and Wnt $5 \mathrm{a} /$ noncanonical signaling in MFH cells may be sufficient to recapitulate the overall pattern of Wnt signaling observed in confluent hMSCs and thus allow for the controlled differentiation of MFH cells into mature connective tissue lineages.

Accordingly, MFH0022 cells were cultured in the presence of both recombinant Wnt2 (rWnt2) and rWnt5a for 72 hours prior to changing medium, and further grown in either ADM or ODM without rWnt5a or rWnt2. Treatment of MFH cells with either ADM or ODM did not result in any significant accumulation of markers of either adipocytic (Figure 6A) or osteogenic (Figure 6B) differentiation. However, MFH cells pretreated with rWnt2 and 


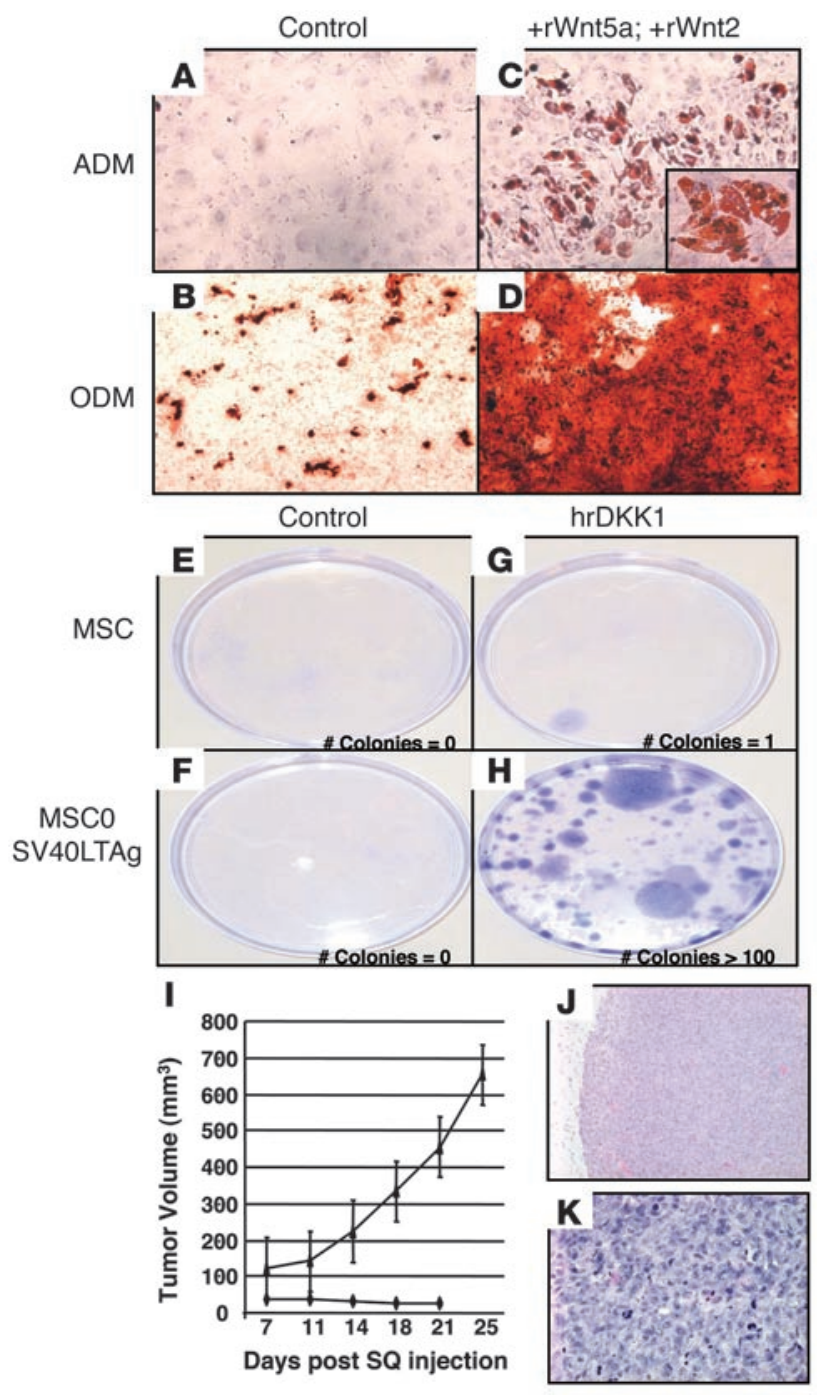

rWnt5a readily accumulated markers of fat and mineralized calcium (Figure 6, C and D, respectively). Notably, the continual exposure of rWnt2 and rWnt5a after the addition of either ADM or ODM to MFH resulted in significantly less accumulation of differentiated cells. Similar treatment with rWnt2 alone resulted in cellular death while treatment with rWnt5a alone led to no evidence of differentiated cells (data not shown).

Inhibition in bMSCs of Wnt-mediated commitment results in transformation and MFH morphology. Having shown that activation of both canonical and noncanonical Wnt signaling is sufficient to commit MFH cells to differentiation, we next sought to test the hypothesis that inhibition of both Wnt signaling pathways in hMSCs may result in tumorigenesis. Since our previous observations suggest that Wnt5a (via Wnt/JNK signaling) is not involved in differentiation but rather mediates a commitment-viability checkpoint, we hypothesized that inhibition of canonical Wnt signaling would be sufficient to block commitment of hMSCs, which may serve as a tumorigenic event. Furthermore, if our initial gene expression analysis experiments were correct in identifying hMSCs as the progenitors of MFH, then the transformed phenotype of hMSCs should resemble MFH. To test this hypothesis, hMSCs and hMSCs previously immortalized with SV40 large T antigen (22) were treated with human recombinant

\section{Figure 6}

Differentiation and transformation via Wnt signaling. MFH cells cultured in ADM (A); ODM (B); ADM, rWnt2, and rWnt5a (C); and ODM, rWnt2, and rWnt5a (D). hMSCs cultured in the absence (E) or presence of hrDkk1 (G); SV40-hMSCLTag cultured in the absence (F) or presence of hrDkk1 $(\mathbf{H})$; tumor volumes at the indicated days of subcutaneously injected SV40-hMSCLTag tumorigenic clones (upper line) and SV40hMSCLTag cells (lower line) as controls into the flanks of Balb/c nude mice. Clones from H\&E staining of tumors formed following subcutaneous injection of in vitro-transformed cells at low (I) and high (J) power. Original magnification, $\times 200 ; \times 400$ (inset).

Dkk1 (hrDkk1) for 2 weeks at a concentration of $50 \mathrm{ng} / \mathrm{ml}$ (based on a previous determination of Dkk1 media levels in expanding hMSCs) (7). As controls, hMSCs and SV40-hMSCs were grown in the absence of Dkk1 supplementation. All cultures were allowed to grow to confluence and then diluted and replated at a low density. Cells were grown for an additional 2 weeks in the presence and/or absence of hrDkk1 or until the detection of colony formation. hMSCs and SV40-hMSCs grown in the presence of hrDkk1 showed tumorigenic colony formation (Figure 6, G and H, respectively). This result was most evident in SV40-hMSCs and not seen in controls. No spontaneous transformation in either hMSCs or SV40-hMSCs grown in the absence of hrDKK1 was observed (data not shown).

To assess the in vivo tumorigenicity of these cells, an SV40-hMSC tumorigenic clone (SV40-hMSC-TC) was isolated and expanded, and $4 \times 10^{6}$ cells were injected subcutaneously into the flanks of nude mice. Parental SV40-hMSC cells were injected for controls. Within 3 weeks, SV40-hMSC-TC formed tumors with average volumes of $454 \mathrm{~mm}^{3}$ in comparison with $24 \mathrm{~mm}^{3}$ for SV40-hMSC parental cells (Figure 6I). Since the current gold standard for diagnosis of MFH is based on tumor morphology, a histological analysis of the formed tumors was performed (3). Histological analysis of SV40-hMSC-TC tumors revealed their high grade and undifferentiated nature (Figure 6, J and K) and their mesenchymal origin, according to IHC studies (vimentin positive; S100, SMA, cytokeratin negative; data not shown). Finally, these tumors were reviewed in a blinded manner by a sarcoma pathologist and morphologically recapitulated MFH histopathology. The only apparent difference between the SV40-hMSC-TC xenograft tumors and human MFH was that the xenograft tumors did not display the full degree of pleomorphism commonly observed in MFH.

\section{Discussion}

MFH as a transformed hMSC. A stem cell-specific gene expression pattern to discriminate between sarcoma subtypes revealed that the profile of hMSCs was significantly associated with that of the MFH but not other sarcoma subtypes. As validation of our postulate on the relationship between MFH and hMSCs, we demonstrated that hMSCs can be transformed into MFH-like tumors using the principles of MSC biology (i.e., Wnt signaling and balance between proliferation and differentiation). This relation of sarcomagenesis to normal mesenchymal differentiation pathways provides fundamental insight into explaining how sarcomas arise (schematically represented, Supplemental Figure 6).

While others have been able to recreate what appear to be highgrade sarcomas by overexpressing known oncogenes or inactivating tumor suppressors (e.g., delivering Cre recombinase to the thighs of mice carrying conditional mutations in K-ras and p53; ref. 23), this study takes into account the normal biology of precursor cells 
TISSUE HOMEOSTASIS

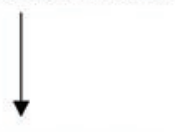

TISSUE TURNOVER

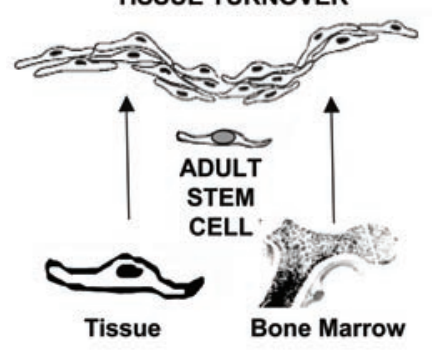

TISSUE DAMAGE

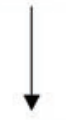

TISSUE REPAIR

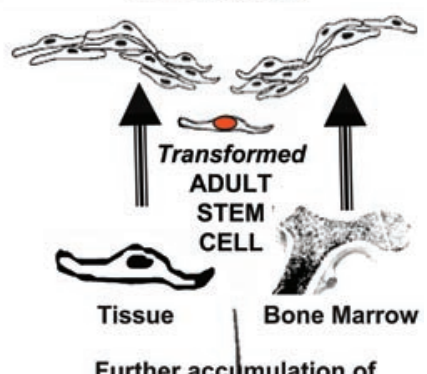

Further accumulation of tumorigenif mutations

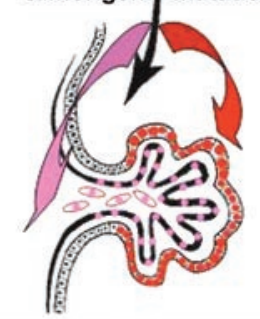

TUMOR DEVELOPMENT H.

to demonstrate how its deregulation results in tumorigenesis. Based on our observations, we propose a model of MFH sarcomagenesis in which a stimulus for hMSC proliferation prior to commitment to differentiation may serve as the backdrop for further genetic changes and tumorigenesis (Figure 7). Specifically, tissue homeostasis is based on the normal turnover of cells that depends on a pool of tissue progenitor cells and/or adult stem cells differentiating into the corresponding mature tissue. Tissue injury/repair may be an even stronger impetus for stem cell recruitment. These states of chronic inflammation (i.e., Crohn disease), chronic infection (i.e., Helicobacter pylori), and chronic physical damage (reflux gastritis) are well associated with the onset of cancer. Thus, failure to commit to differentiation (through inability to activate Wnt signaling as discussed in this manuscript for hMSCs) may result in a pool of hyperproliferating stem cells with enhanced tumorigenic potential. These cells may then secondarily become deficient in response to damage, have enhanced proliferation, and generate antiapoptotic signals, ultimately resulting in the ability to generate a complete cancer initiating unit. Finally, although this model is potentially equally applicable to any tissue, in light of our observations that hMSCs when transformed assume a MFH morphology and IHC profile, it should be pointed out that $\mathrm{MFH}$ of bone is a well-characterized entity and has been reported to occur as a postarthroplastic complication (24).

Wnt signaling in bMSCs and MFH. A critical observation from this study is the role of $\mathrm{Wnt} 2 /$ canonical signaling in the "commitment" of hMSCs to differentiation, as defined by its control over the accumulation of EMofMD. Another significant finding is that Wnt5/noncanonical (via JNK) signaling appears to control a viability checkpoint at the time of hMSC commitment. The concomitant activation of both pathways appears necessary and sufficient to commit hMSCs to differentiation in the presence of specific hMSC

\section{Figure 7}

Schematic diagram illustrating a working hypothesis regarding tumorigenesis. Enhanced recruitment of adult stem cells and/or tissue precursors under conditions that result in tissue damage may promote development of the first oncogenic event that predisposes that cell to accumulate further genetic damage, ultimately resulting in tumor initiation and progression. See text for details.

maturation media. EMofMD can be detected in MFH cells in which the canonical pathway is activated (via Wnt2), but this is insufficient for terminal differentiation, suggesting that there must be crosstalk between the canonical and noncanonical Wnt pathways during normal commitment to a mature mesenchymal cell.

Wnt signaling as a tumor suppressor. An unexpected finding in this study is the observation that inhibition of Wnt signaling (via continuous culturing in the presence of hrDKK1) is itself pro-oncogenic or, conversely, that active Wnt signaling serves a tumor suppressor function in hMSCs. This conclusion is based on our observations that culturing of hMSCs and SV40-hMSC in the presence of hrDkk1 results in acquisition of clonogenicity in vitro and tumors in nude mice. These results are strikingly different from the role of canonical Wnt signaling in carcinomas, where activating mutations of several genes within this pathway have been reported to be tumorigenic. Nuclear $\beta$-catenin accumulation as an indicator of active canonical Wnt signaling is a well-documented event in colorectal (25), prostate (26), breast (27), and ovarian carcinomas (28) as well as melanomas (29). Additionally, numerous stabilizing mutations in $\beta$-catenin (30) as well as inactivating mutations of members of the APC/AXIN/ GSK-3 $\beta$ complex $(31,32)$ have been well characterized in colorectal carcinomas. Furthermore, downregulation of $\beta$-catenin in colorectal carcinoma cell lines leads to loss of proliferative capacity (33), thus suggesting that aberrant Wnt/ $\beta$-catenin is not just a marker but also contributes to the malignant phenotype.

In contrast with carcinomas, no Wnt/ $\beta$-catenin nuclear activity has been noted in sarcomas (34) with the exception of desmoid tumors (deep fibromatoses) (35) and synovial sarcomas (36). Taken together, these data suggest that Wnt signaling may limit development of mesenchymal tumors and thus have a very different role in sarcomagenesis as compared with carcinogenesis. Supporting this notion is the observation that Wnt signaling may have a different role in epithelial and mesenchymal development. Epithelial-mesenchymal transition is an important process during development by which epithelial cells acquire mesenchymal or fibroblast-like properties and show reduced intercellular adhesions and increased motility mediated by active Wnt/ $\beta$-catenin signaling. In contrast, mesenchymal-epithelial transition is observed as Wnt/ $\beta$-catenin is downregulated (37). Thus, Wnt signaling mediates development of and/or commitment to the mesenchymal program, a conclusion supported by the observation that stabilized $\beta$-catenin in the mouse zygote leads to premature epithelial-mesenchymal transition in the epiblast (38). Although our data further support a role of Wnt signaling in committing mesenchymal progenitor cells to mesenchymal differentiation and mesenchymal tissue development, the role of Wnt signaling after commitment (i.e., after the pluripotent progenitor stage) appears to be both lineage specific and even temporally dependent. For progenitor cells committed to adipogenesis, Wnt/ $\beta$-catenin signaling is inhibitory $(34,38)$. Conversely, for progenitor cells committed to the osteogenic lineage, Wnt signaling promotes development (39). In an even more complex example, Wnt signaling in developing cardiomyocytes 
acts in phases where it must be activated, then repressed, and later activated again (40). Finally, it should be pointed out that others (41) have reported that Wnt3a signaling is inhibitory to hMSC differentiation. The differences in these results suggest that interactions between specific Wnts and Wnt receptors (i.e., frizzled family) may result in potential activation of different downstream pathways (42). The differential effect of Wnt/ $\beta$-catenin activation has been demonstrated even on specific $\beta$-catenin target genes that are either activated or repressed based on context (e.g., Sox 9; refs. 43, 44). Thus, our findings that c-myc and cyclin D1 levels inversely correlate with $\beta$-catenin activity in both hMSCs and MFH are further supported by our data regarding the primed repressive state or bivalent chromatin structure $(45,46)$ (Supplemental Text) of these genes at the point of commitment. Together, these data suggest that $\beta$-catenin directly represses these genes in confluent hMSCs and MFH cells in which hMSC Wnt signaling patterns are recapitulated. Finally, at least as may apply to the hMSC state, this tumorsuppressor role for specific Wnt2 signaling may mediate differentiation via activation of $\beta$-catenin in conjunction with Wnt5a/JNK.

In conclusion, data from this study establish a relationship between adult stem cells and their transformation to a specific sarcoma subtype, providing a previously missing link in the relationship between stem cells and cellular transformation. This paradigm shift sets the stage for further exploration and modeling of adult onset tumorigenesis. Results presented also uncover what we believe is a novel tumor suppression role for Wnt signaling in solid tumors. Finally, by elucidating key signaling patterns in hMSCs at the point of commitment and then reestablishing these patterns in tumor cells, we demonstrate how solid tumors could be reprogrammed to resume normal differentiation.

\section{Methods}

Cell culture, retroviral transduction, siRNA treatment, and cell cycle analysis. hMSCs were kindly provided by Darwin Prockop (Tulane University, New Orleans, Louisiana, USA) and propagated in continuous culture medium (20\% FBS, Atlanta Bio in DMEM, Gibco BRL). Production of retroviral supernatants was carried out by calcium phosphate-mediated transfection of amphotropic packaging Phoenix cells. High-titer retroviral supernatants were passed through a $0.45-\mu \mathrm{m}$ filter and supplemented with $6 \mu \mathrm{g} / \mathrm{ml}$ polybrene (Sigma-Aldrich). siRNAs for Wnt2 (Hs_WNT2_1), Wnt5a (Hs_WNT5A_7), and Dkk1 (Hs_DKK1_1) were purchased from QIAGEN (Xeragon) and transfected using the HiPerFect Transfection Reagent (QIAGEN).

Gene expression analysis. Hierarchical cluster analysis was performed using Pearson's correlation coefficient. Gene ranking per subtype was performed using ANOVA (parametric test, Benajmini and Hochberg false discovery rate, $P<0.05$; and Student-Neuman-Keuls post hoc analysis). RNA from soft tissue sarcoma samples was hybridized to HG U133A oligonucleotide arrays per standard protocols of the MSKCC Genomics Core Facility (described in detail in ref. 9).

Protein isolation and immunoblot analysis. Immunoblot assays were performed on $25 \mu \mathrm{g}$ of total protein extracted from proliferating hMSCs and MFH0022 as previously described (47). Blots were probed with antibodies directed against the following: human Dkk1 (PoAb-Dkk1) (R\&D Human Affinity Purified Polyclonal Ab, AF1096); total $\beta$-catenin (Upstate, AB19022); active $\beta$-catenin (Upstate, anti-ABC, clone 8E7, 05-665); Wnt2 (Santa Cruz H-20, SC5208); Wnt5a (Santa Cruz H-58, SC30244); LEF1 (Santa Cruz N-17, SC8519); cyclin D1 (Abcam 24249); c-myc (Santa Cruz $\mathrm{N}-262, \mathrm{SC764})$; and $\beta$-tubulin (Upstate). Secondary anti-mouse, antigoat, or anti-rabbit (Amersham) antibodies were used to visualize proteins using an ECL detection system (Amersham). Ideal concentrations for each antibody were empirically determined using company-recommended controls. Working concentrations ranged from 1:500 to 1:2000 dilutions of recommended stock solutions.

Immunohistochemistry. Tissue sections were deparaffinized, treated with $1 \% \mathrm{H}_{2} \mathrm{O}_{2}$, immersed in $10 \mathrm{mM}$ boiling citrate buffer for 15 minutes, and incubated in $10 \%$ normal horse serum for 30 minutes at room temperature. Samples were incubated with antibodies against CD54 (clone 28, BD Biosciences - Pharmingen), hepatocyte growth factor (clone 24612.111, AbCam), muscle actin (clone HHF35, AbCam), $\alpha$-smooth muscle actin (clone A2547, Sigma-Aldrich), adiponectin (anti-Acrp30 antiserum, AbCam), leptin (ab2125, AbCam), and Dkk1 (AF1096, R\&D). Samples were further incubated with biotinylated anti-mouse, anti-rabbit, or anti-goat antibodies (Vector Laboratories) and then followed by avidin-biotin peroxidase complexes (Vector Laboratories) for $30 \mathrm{~min}$ utes. Diaminobenzidine was used as the chromogen and hematoxylin as the nuclear counter stain.

Protein activity assays. $\beta$-catenin activity was measured via the ELISA Human Total $\beta$-catenin DuoSet IC (R\&D, DYC1329E) per the manufacturer's guidelines, except for the substitution of anti-active $\beta$-catenin (anti-ABC, clone 8E7 Upstate) as the capture antibody. Wnt/calcium pathway activity was measured via Protein Kinase C Activity Kit (Stress Bioreagents, EKS-420A) per the manufacturer's guidelines, except for the preliminary step in which the cell lysates were immunoprecipitated with pan PKC antibody (SC-10800, Santa Cruz Biotechnology Inc.). Wnt/ Rho GTPase pathway was measured via the ROK $\alpha /$ ROCK-II KinEAS FP-645nm FarRed Assay (Upstate, 32-118) according to the manufacturer's instructions. Wht/JNK pathway activity was measured via JNK Activity Kit (Sigma-Aldrich, CS0380) performed according to the manufacturer's instructions. Absorbance measurements were performed using the Victor V Plate Reader (PerkinElmer).

In vitro and in vivo tumorigenic assays. hMSCs and hMSCs previously immortalized with SV40-large T antigen (SV40-hMSC) (22) were treated with hrDKK1 (R\&D) for 2 weeks at a concentration of $50 \mathrm{ng} / \mathrm{ml}$, based on a previous determination of endogenous Dkk1 media levels in expanding hMSCs (7). As controls, hMSCs and SV40-hMSC were grown in the absence of Dkk1 supplementation. All cultures were allowed to grow to confluence, then diluted and replated at a density of $1000 \mathrm{cells} / 10 \mathrm{~cm}$ plate. Cells were grown for an additional 2 weeks in the presence and/or absence of hrDKK1 or until colony formation was observed, at which point all plates were stained with crystal violet. To assess the in vivo tumorigenicity of in vitrogenerated clones, an SV40-hMSC tumorigenic clone (SV40-hMSC-TC) was isolated and expanded, and $4 \times 10^{6}$ cells were injected subcutaneously into the flanks of Balb/c nude mice. Parental SV40-hMSC cells were injected as controls. Mice were observed for 2 weeks and then sacrificed. Tumors were excised and analyzed by $\mathrm{H} \& \mathrm{E}$ staining. Animals were housed in pathogen-free housing and all experiments were approved and strictly subjected to guidelines as dictated by the Institutional Animal Care and Use Committee and the Research Animal Resource Center at Memorial Sloan-Kettering Cancer Center.

Differentiation assays. To induce adipocytic differentiation, MFH0022 cells were grown in DMEM/F12 supplemented with $0.5 \mu \mathrm{M}$ dexamethasone, $0.5 \mu \mathrm{M}$ isobutylmethylxanthine, and $50 \mu \mathrm{M}$ indomethacin for 21 days. To induce osteogenic differentiation, MFH0022 cells were grown in DMEM/F12 supplemented with $10^{-8} \mathrm{M}$ dexamethasone, $5 \mathrm{mM} \beta$-glycerophosphate, and $50 \mu \mathrm{g} / \mathrm{ml} \mathrm{L}$-ascorbic acid for 21 days. Human recombinant Wnt2 and mouse recombinant Wnt5a (both from R\&D Systems) were used at $100 \mathrm{ng} / \mathrm{ml}$. Oil red O (Sigma-Aldrich) and Alizarin red -S (Sigma-Aldrich) staining was performed as previously described (7). Additionally, MFH0022 cells were grown in the presence or absence of rhDKK1, as indicated in Results. The differentiation protocol utilized 
represents a modification of the hMSC differentiation protocol outlined in the Supplemental Text.

\section{Acknowledgments}

We thank Gary K. Schwartz for his critical input to the project and reading of the manuscript. We would like to thank Darwin Prockop and the Tulane University Gene Center for providing us with hMSCs as well as detailed instructions regarding their propagation and differentiation. We greatly appreciate the use of the MFH0022 and LS141 cell lines both generated from human tissue and kind gifts of Sam Singer, MSKCC. We would also like to thank Maria E. Dudas for assisting with immunohistochemistry and Agnes Viale, Genomics Core Facility, for assistance with U133A Affymetrix array processing. I. Matushanksy receives support from the American Cancer Society and the Clinical Scholars/Charles Revson Foundation. R.G. Maki receives research support from NCI Program project CA47179, Spin4survival.org, and The Shuman Family Fund for GIST Research. C. Cordon-Cardo receives support from NCI Program Project CA47179 and philanthropic funds.

Received for publication December 29, 2006, and accepted in revised form August 3, 2007.

Address correspondence to: Igor Matushansky, Department of Medicine, Columbia University, 1130 St. Nicholas Ave., Room 301, New York, New York 10032, USA. Phone: (212) 851-4556; Fax: (212) 851-4550; E-mail: im17@columbia.edu. Or to: Robert G. Maki, Department of Medicine, Memorial Sloan-Kettering Cancer Center, 1275 York Ave., Box 223, New York, New York 10065 , USA. Phone: (212) 639-5720; Fax: (646) 422-2076; E-mail: makir@ mskcc.org. Or to: Carlos Cordon-Cardo, Department of Pathology, Columbia University, 1130 St. Nicholas Ave., Room 309, New York, New York 10032, USA. Phone: (212) 851-4554; Fax: (212) 851-4550; E-mail: cc2791@columbia.edu.
1. Ozzello, L., Stout, A.P., and Murray, M.R. 1963. Cultural characteristics of malignant histiocytomas and fibrous xanthomas. Cancer. 16:331-344.

2. Taxy, J.B., and Battifora, H. 1977. Malignant fibrous histiocytoma. An electron microscopic study. Cancer. 40:254-267.

3. Fletcher, C.D. 2006. The evolving classification of soft tissue tumours: an update based on the new WHO classification. Histopathology. 48:3-12.

4. Sarcoma Meta-analysis Collaboration. 1997. Adjuvant chemotherapy for localised resectable soft-tissue sarcoma of adults: meta-analysis of individual data. Sarcoma Meta-analysis Collaboration. Lancet. 350:1647-1654.

5. Sarcoma Meta-analysis Collaboration. 2000. Adjuvant chemotherapy for localised resectable soft tissue sarcoma in adults. Sarcoma Meta-analysis Collaboration (SMAC). Cochrane Database Syst. Rev. 2:CD001419.

6. Fedi, P., et al. 1999. Isolation and biochemical characterization of the human Dkk-1 homologue, a novel inhibitor of mammalian Wnt signaling. J. Biol. Chem. 274:19465-19472.

7. Gregory, C.A., Singh, H., Perry, A.S., and Prockop, D.J. 2003. The Wnt signaling inhibitor dickkopf-1 is required for reentry into the cell cycle of human adult stem cells from bone marrow. J. Biol. Chem. 278:28067-28078.

8. Willert, K., and Jones, K.A. 2006. Wnt signaling: is the party in the nucleus? Genes Dev. 20:1394-1404

9. Segal, N.H., et al. 2003. Classification and subtype prediction of adult soft tissue sarcoma by functional genomics. Am. J. Pathol. 163:691-700.

10. Eckfeldt, C.E., Mendenhall, E.M., and Verfaillie, C.M. 2005. The molecular repertoire of the 'almighty' stem cell. Nat. Rev. Mol. Cell Biol. 6:726-737.

11. Rao, R.R., and Stice, S.L. 2004. Gene expression profiling of embryonic stem cells leads to greater understanding of pluripotency and early developmental events. Biol. Reprod. 71:1772-1778.

12. Gazziola, C., et al. 2003. Malignant fibrous histiocytoma: a proposed cellular origin and identification of its characterizing gene transcripts. Int. J. Oncol. 23:343-351.

13. Barberi, T., Willis, L.M., Socci, N.D., and Studer, L. 2005. Derivation of multipotent mesenchymal precursors from human embryonic stem cells. PLoS Med. 2:e161.

14. Baird, K., et al. 2005. Gene expression profiling of human sarcomas: insights into sarcoma biology. Cancer Res. 65:9226-9235.

15. Gregory, C.A., et al. 2005. Dkk-1-derived synthetic peptides and lithium chloride for the control and recovery of adult stem cells from bone marrow. J. Biol. Chem. 280:2309-2323.

16. Etheridge, S.L., Spencer, G.J., Heath, D.J., and
Genever, P.G. 2004. Expression profiling and functional analysis of wnt signaling mechanisms in mesenchymal stem cells. Stem Cells. 22:849-860.

17. Veeman, M.T., Axelrod, J.D., and Moon, R.T. 2003. A second canon. Functions and mechanisms of beta-catenin-independent Wnt signaling. Dev. Cell. 5:367-377.

18. Gonindard, C., et al. 1997. Synthetic hispidin, a PKC inhibitor, is more cytotoxic toward cancer cells than normal cells in vitro. Cell Biol. Toxicol. 13:141-153.

19. Kusama, K., et al. 2003. Deactivation of ROCK-II by Y-27632 enhances basolateral pancreatic enzyme secretion and acute pancreatitis induced by CCK analogues. Biochem. Biophys. Res. Commun. 305:339-344.

20. Bennett, B.L., et al. 2001. SP600125, an anthrapyrazolone inhibitor of Jun N-terminal kinase. Proc. Natl. Acad. Sci. U. S. A. 98:13681-13686.

21. Yu, C., et al. 2004. JNK suppresses apoptosis via phosphorylation of the proapoptotic Bcl-2 family protein BAD. Mol. Cell. 13:329-340.

22. Nishioka, K., et al. 2003. Immortalization of bone marrow-derived human mesenchymal stem cells by removable simian virus $40 \mathrm{~T}$ antigen gene: analysis of the ability to support expansion of cord blood hematopoietic progenitor cells. Int. J. Oncol. 23:925-932.

23. Kirsch, D.G., Dinulescu, D.M., Santiago, P.M., and Jacks, T. 2005. A mouse model for soft tissue sarcoma. In 2005 ASCO Annual Meeting Proceedings. June 1, 2005. J. Clin. Oncol. 23(Suppl. 16S):9014.

24. Lucas, D.R., Miller, P.R., Mott, M.P., Kronick, J.L., and Unni, K.K. 2001. Arthroplasty-associated malignant fibrous histiocytoma: two case reports. Histopathology. 39:620-628.

25. Rubinfeld, B., et al. 1993. Association of the APC gene product with beta-catenin. Science. 262:1731-1734.

26. Voeller, H.J., Truica, C.I., and Gelmann, E.P. 1998. Beta-catenin mutations in human prostate cancer. Cancer Res. 58:2520-2523.

27. Colter, D.C., et al. 2000. Rapid expansion of recycling stem cells in cultures of plastic-adherent cells from human bone marrow. Proc. Natl. Acad. Sci. U. S. A. 97:3213-3218

28. Kobayashi, K., Sagae, S., Nishioka, Y., Tokino, T., and Kudo, R. 1999. Mutations of the beta-catenin gene in endometrial carcinomas. Jpn. J. Cancer Res. 90:55-59.

29. Rubinfeld, B., et al. 1997. Stabilization of betacatenin by genetic defects in melanoma cell lines. Science. 275:1790-1792.

30. Ilyas, M., Tomlinson, I.P., Rowan, A., Pignatelli, M., and Bodmer, W.F. 1997. Beta-catenin mutations in cell lines established from human colorectal cancers. Proc. Natl. Acad. Sci. U. S. A. 94:10330-10334.

31. Kinzler, K.W., et al. 1991. Identification of a gene located at chromosome $5 \mathrm{q} 21$ that is mutated in colorectal cancers. Science. 251:1366-1370.

32. Kinzler, K.W., and Vogelstein, B. 1996. Lessons from hereditary colorectal cancer. Cell. 87:159-170.

33. Green, D.W., Roh, H., Pippin, J.A., and Drebin, J.A. 2001. Beta-catenin antisense treatment decreases beta-catenin expression and tumor growth rate in colon carcinoma xenografts. J. Surg. Res. 101:16-20.

34. Arango, N.A., et al. 2005. Conditional deletion of beta-catenin in the mesenchyme of the developing mouse uterus results in a switch to adipogenesis in the myometrium. Dev. Biol. 288:276-283.

35. Bhattacharya, B., et al. 2005. Nuclear beta-catenin expression distinguishes deep fibromatosis from other benign and malignant fibroblastic and myofibroblastic lesions. Am. J. Surg. Pathol. 29:653-659.

36. Saito, T., et al. 2002. APC mutations in synovial sarcoma. J. Pathol. 196:445-449.

37. Larue, L., and Bellacosa, A. 2005. Epithelialmesenchymal transition in development and cancer: role of phosphatidylinositol $3^{\prime}$ kinase/AKT pathways. Oncogene. 24:7443-7454.

38. Kemler, R., et al. 2004. Stabilization of beta-catenin in the mouse zygote leads to premature epithelialmesenchymal transition in the epiblast. Development. 131:5817-5824

39. Hartmann, C. 2006. A Wnt canon orchestrating osteoblastogenesis. Trends Cell Biol. 16:151-158.

40. Foley, A., and Mercola, M. 2004. Heart induction: embryology to cardiomyocyte regeneration. Trends Cardiovasc. Med. 14:121-125.

41. Boland, G.M., Perkins, G., Hall, D.J., and Tuan, R.S. 2004. Wnt 3a promotes proliferation and suppresses osteogenic differentiation of adult human mesenchymal stem cells. J. Cell. Biochem. 93:1210-1230.

42. Mikels, A.J., and Nusse, R. 2006. Purified Wnt5a protein activates or inhibits beta-catenin-TCF signaling depending on receptor context. PLoS Biol. 4:e115.

43. Blache, P., et al. 2004. SOX9 is an intestine crypt transcription factor, is regulated by the Wnt pathway, and represses the CDX2 and MUC2 genes. J. Cell Biol. 166:37-47.

44. Hill, T.P., Spater, D., Taketo, M.M., Birchmeier, W., and Hartmann, C. 2005. Canonical Wnt/betacatenin signaling prevents osteoblasts from differentiating into chondrocytes. Dev. Cell. 8:727-738.

45. Bernstein, B.E., et al. 2006. A bivalent chromatin structure marks key developmental genes in embryonic stem cells. Cell. 125:315-326.

46. Spivakov, M., and Fisher, A.G. 2007. Epigenetic signatures of stem-cell identity. Nat. Rev. Genet. 8:263-271.

47. Matsushime, H., et al. 1994. D-type cyclin-dependent kinase activity in mammalian cells. Mol. Cell. Biol. 14:2066-2076. 